\title{
Efecto de la suplementación con DHA durante el embarazo en depresión materna y neurodesarrollo de niños jóvenes: un estudio randomizado controlado (1)
}

\author{
Makrides M, Gibson RA, McPhee AJ, Yelland L, Quinlivan J, Ryan P; DOMInO \\ Investigative Team. Effect of DHA supplementation during pregnancy on maternal de- \\ pression and neurodevelopment of young children: a randomized controlled trial. JAMA \\ 2010;304(15):1675-83.
}

Análisis crítico: Jorge Carvajal C. PhD, Claudio Vera PG. MSc.

Unidad de Medicina Materno Fetal, División de Obstetricia y Ginecología, Facultad de Medicina,

Pontificia Universidad Católica de Chile.

\section{RESUMEN (1)}

Antecedentes: No existe certeza respecto de los beneficios de la suplementación dietaria con ácido docosahexanoico (DHA) para la mujer embarazada y su hijo, pese a la recomendación internacional de aumentar la ingesta de DHA en el embarazo. Objetivo: Determinar si el incremento de DHA durante la última mitad del embarazo resultará en menos mujeres con altos niveles de depresión y mejorará el neurodesarrollo de su hijo. Método: Estudio doble ciego, multicéntrico, randomizado controlado (DHA para Optimizar Resultado Materno Infantil [DOMInO]) en 5 maternidades australianas de 2399 mujeres con embarazos únicos, menores a 21 semanas, quienes fueron reclutadas entre el 31 de octubre de 2005 y el 11 de enero de 2008. El seguimiento de los niños $(n=726)$ fue completado hasta el 16 de diciembre de 2009. Intervención: Cápsulas de aceite de pescado ricas en ácido docosahexanoico (800 mg/d de DHA) o cápsulas de aceite vegetal sin DHA desde el ingreso al estudio hasta el parto. Principal medida de resultado: altos niveles de síntomas depresivos en las mujeres, según puntaje mayor a 12 en la escala de Edimburgo de Depresión Post Natal a las 6 semanas y 6 meses posparto. Desarrollo cognitivo y del lenguaje en niños, medidos por la escala de Bayley para desarrollo infantil y del niño, a los 18 meses. Resultados: De las
2399 mujeres enroladas, 96,7\% completaron el estudio. El porcentaje de mujeres con altos niveles de síntomas depresivos durante los primeros 6 meses posparto no fue diferente entre el grupo DHA y el grupo control $(9,67 \%$ vs. $11,9 \%$, RR ajustado 0,85 ; IC95\% 0,70-1,02; $p=0,09$ ). Puntajes promedio de resultado cognitivo (diferencia promedio ajustada $0,01$; IC $95 \% 1,36-1,37 ; p=0,99)$ y puntaje promedio de resultado compuesto de lenguaje (diferencia promedio ajustada 1,42; IC95\% 3,07-0,22; $p=0,09$ ) no fue diferente entre los grupos DHA y control. Conclusión: El uso de cápsulas de aceite de pescado rico en DHA comparado con cápsulas de aceite vegetal durante el embarazo, no resulta en disminución de los niveles de depresión posparto en las madres o mejoría del desarrollo cognitivo y del lenguaje en sus hijos durante la infancia temprana.

\section{ANALISIS DE LA INVESTIGACIÓN}

\section{A. Relevancia clínica de la investigación}

Uno de los objetivos del control prenatal es definir las indicaciones de suplementación nutricional en la embarazada. Estas recomendaciones debieran estar basadas en la mejor evidencia disponible. Por ejemplo, la suplementación universal con hierro ha demostrado disminuir el riesgo de anemia al término, sin embargo, la evidencia disponible no muestra 
beneficio en reducción de eventos adversos clínicamente relevantes para la madre o el recién nacido (2). Esta situación ha llevado a replantear su dosis y recomendación, dependiendo del contexto clínico $(2,3)$. Lo anterior resalta la necesidad de la evaluación apropiada de cualquier suplementación durante el embarazo y su contexto antes de recomendar su uso.

Los ácidos grasos omega-3, especialmente el ácido docosahexaenoico (DHA), han sido propuestos como un suplemento nutricional útil para la embarazada, esperando lograr reducir la incidencia de preeclampsia o parto prematuro, o intentando mejorar la lactancia materna, el desarrollo neurológico infantil y evitar la depresión materna.

En los últimos años ha existido una fuerte presión de la industria farmacéutica para agregar suplementos nutricionales, como el DHA, a la embarazada, sin embargo, la evidencia que sustenta su uso deriva de estudios observacionales o randomizados, con serios problemas metodológicos. La revisión sistemática de la base de datos Cochrane del año 2006, sugiere que la suplementación con DHA durante el embarazo, no es de utilidad para reducir el riesgo de preeclampsia, parto prematuro, bajo peso de nacimiento o restricción de crecimiento fetal (4). Persiste la duda respecto de la utilidad del DHA en reducir el riesgo de depresión o mejorar el neurodesarrollo del niño.

\section{B. El estudio (1)}

Diseño: Investigación clínica controlada aleatorizada, doble ciego, en 5 centros australianos. Pacientes: Mujeres con embarazos únicos menor de 21 semanas. Criterios de exclusión: Estar usando DHA como suplemento prenatal, feto con anomalía con- génita mayor, ser portadora de un trastorno de la coagulación en el cual se ha contraindicado el aceite de pescado, uso de anticoagulantes, abuso de drogas o alcohol, estar participando en otro estudio de ácidos grasos, ser incapaz de dar el consentimiento informado o no hablar inglés como lenguaje primario. Intervención: Tres cápsulas diarias de 500 $\mathrm{mg}$ de concentrado de aceite de pescado rico en DHA, logrando una dosis de $800 \mathrm{mg} /$ día de DHA y $100 \mathrm{mg} / \mathrm{día}$ de ácido eicosapentaenoico. Control: Tres cápsulas diarias de $500 \mathrm{mg}$ de aceite vegetal, que no contiene DHA. Ambos administrados desde las 20 semanas hasta el parto. Resultado primario evaluado: Se evaluaron dos resultados primarios: 1 . Altos niveles de síntomas depresivos a las 6 semanas y 6 meses posparto, medido a través de autoadministración de la escala de Edimburgo de depresión posnatal; 2. Neurodesarrollo a los 18 meses de edad medido por la escala de Bayley para desarrollo cognitivo y de lenguaje en niños e infantes, tercera edición, BSID-III. Los síntomas de depresión fueron medidos en todas las mujeres, $y$ el neurodesarrollo en todos los prematuros y en una muestra seleccionada al azar de niños de término, en ambas ramas. Resultados secundarios evaluados: Duración del embarazo, parto antes de las 37 o 34 semanas, inducción o cesárea en embarazo de postérmino, tasa de cesárea, pérdida de sangre durante el parto, hemorragia posparto, peso de nacimiento promedio y bajo peso al nacer. A los 18 meses se midió: hospitalización o muerte materna, hospitalización, malformación o muerte infantil. Resultados: No hubo diferencias significativas en el resultado primario (Tabla I). Si hubo diferencias significativas en algunos resultados secundarios (Tabla I).

Tabla I

RESULTADOS PRIMARIO Y SECUNDARIOS DE LA INVESTIGACIÓN

\begin{tabular}{lccc}
\hline Resultado primario & DHA & Placebo & Medidas de efecto ajustadas (IC 95\%) \\
\hline Altos niveles de síntomas depresivos & $9,67 \%$ & $11,9 \%$ & RR (IC 95\%) y valor $\mathrm{p}$ \\
& & & $0,85(0,70$ a 1,02$) ; \mathrm{p}=0,09$ \\
Alteraciones del neurodesarrollo & & & Diferencia media (IC 95\%) y valor $\mathrm{p}$ \\
$\quad$ - Puntaje promedio cognitivo & 101,81 & 101,75 & $0,01(-1,36$ a 1,37$) ; \mathrm{p}=0,99$ \\
- Puntaje promedio lenguaje & 96,47 & 97,94 & $-1,42(-3,07$ a 0,22$) ; \mathrm{p}=0,09$ \\
Resultados secundarios & & & RR, o diferencia media (IC 95\%) \\
Parto < 34 semanas & $1,09 \%$ & $2,25 \%$ & $0,49(0,25$ a 0,94$)$ \\
Postérmino & $17,59 \%$ & $13,72 \%$ & $1,28(1,06$ a 1,54$)$ \\
Peso de nacimiento & $3475 \mathrm{~g}$ & $3407 \mathrm{~g}$ & $68(23$ a 114$)$ \\
Bajo peso al nacer & $3,41 \%$ & $5,27 \%$ & $0,65(0,44$ a 0,96$)$ \\
Hospitalización Infantil & $1,75 \%$ & $3,08 \%$ & $0,57(0,34$ a 0,97$)$ \\
\hline
\end{tabular}




\section{Análisis crítico}

Validez interna: El estudio aporta conocimiento relevante para un tema de activa discusión. El diseño es apropiado a la pregunta del estudio. Se explica claramente el cálculo de tamaño muestral, y este fue cumplido. La randomización fue adecuada, el ocultamiento de la secuencia de randomización fue resguardado. La intervención fue ciega. El análisis se efectuó bajo el principio de intención de tratar. El seguimiento fue completo. Existió planificación en el análisis con datos faltantes. No se detuvo precozmente por beneficio. Comentario: No hemos encontrado problemas metodológicos en la investigación que hagan sospechar la presencia de sesgo. La principal limitación es el menor número de eventos en el control, que lo estimado en el cálculo de tamaño muestral inicial, dejando al estudio con menor poder y mayor riesgo de error tipo II (no encontrar beneficio cuando existe). Aún así, los autores estiman que tiene poder adecuado para detectar diferencias clínicamente relevantes de un $4 \%$, en el evento de interés materno primario. Conclusión: Estudio con bajo riesgo de sesgo que no muestra beneficio significativo de suplementación con DHA durante el embarazo, para prevenir síntomas de depresión posparto o mejorar el neurodesarrollo infantil. Por lo cual, no apoya la suplementación rutinaria de DHA en embarazadas para esos eventos de interés. Resta por definir el potencial rol del DHA en lograr mejoría de otros parámetros perinatales importantes, como parto prematuro o bajo peso al nacer.

\section{REFERENCIAS}

1. Makrides M, Gibson RA, McPhee AJ, Yelland L, Quinlivan J, Ryan P; DOMInO Investigative Team. Effect of DHA supplementation during pregnancy on maternal depression and neurodevelopment of young children: a randomized controlled trial. JAMA 2010;304(15):1675-83.

2. Peña-Rosas JP, Viteri FE. Effects and safety of preventive oral iron or iron+folic acid supplementation for women during pregnancy. Cochrane Database Syst Rev 2009;(4):CD004736.

3. National collaborating centre for women's and children's health. Antenatal Care: Rutine Care for the Healthy Pregnant Woman. London: RCOG Press, 2008.

4. Makrides M, Duley L, Olsen SF. Marine oil, and other prostaglandin precursor, supplementation for pregnancy uncomplicated by pre-eclampsia or intrauterine growth restriction. Cochrane Database Syst Rev 2006;3:CD003402. 